\title{
EVERETTIAN QUANTUM MECHANICS AND PHYSICAL PROBABILITY: AGAINST THE PRINCIPLE OF "STATE SUPERVENIENCE"
}

\author{
Lina Jansson*
}

\begin{abstract}
Everettian quantum mechanics faces the challenge of how to make sense of probability and probabilistic reasoning in a setting where there is typically no unique outcome of measurements. Wallace has built on a proof by Deutsch to argue that a notion of probability can be recovered in the many worlds setting. In particular, Wallace argues that a rational agent has to assign probabilities in accordance with the Born rule. This argument relies on a rationality constraint that Wallace calls state supervenience. I argue that state supervenience is not defensible as a rationality constraint for Everettian agents unless we already invoke probabilistic notions.
\end{abstract}

idajansson@ntu.edu.sg

\author{
Nanyang Technological University \\ Philosophy Programme \\ 14 Nanyang Drive
}

Singapore 637332

\footnotetext{
${ }^{\star}$ Thank you to Wayne Myrvold for discussions of very early ideas in this paper in the "Many-Worlds Interpretation of Quantum Mechanics" seminar at the University of Michigan. Thanks to Dave Baker and Dan Peterson for many helpful discussions in the same seminar. A particular thanks to Chip Sebens for helpful comments on an earlier draft. Thank you also to the audiences at the 2013 AAP and the 2014 NUS workshop in formal epistemology for their comments, suggestions, and objections. Finally, thank you to the members of the Nottingham reading group. This work was partially supported by a grant from Nanyang Technological University and a visiting fellowship from the University of Nottingham.
} 


\title{
EVERETTIAN QUANTUM MECHANICS AND PHYSICAL PROBABILITY: AGAINST THE PRINCIPLE OF "STATE SUPERVENIENCE"
}

\begin{abstract}
Everettian quantum mechanics faces the challenge of how to make sense of probability and probabilistic reasoning in a setting where there is typically no unique outcome of measurements. Wallace has built on a proof by Deutsch to argue that a notion of probability can be recovered in the many worlds setting. In particular, Wallace argues that a rational agent has to assign probabilities in accordance with the Born rule. This argument relies on a rationality constraint that Wallace calls state supervenience. I argue that state supervenience is not defensible as a rationality constraint for Everettian agents unless we already invoke probabilistic notions.
\end{abstract}

\section{INTRODUCTION}

Everettian quantum mechanics has many virtues, and in particular it offers a strikingly simple solution to the measurement problem. The general outline of how the theory does so is by now familiar. Instead of trying to find some means by which the formalism can be supplemented in order to guarantee that there is only one determinate outcome of measurements, the theory claims that there is, typically, no such thing as the result of a measurement (or indeed of an interaction in general). Rather, all outcomes that are allowed by the formalism really occur, but they do so on separate, relatively isolated, branches of the universe. ${ }^{1}$

However, the theory faces a peculiar challenge when it comes to making sense of the notion of probability and probabilistic notions of confirmation. In particular, it is hard to make sense of a probability assignment other than 1 or 0 within the theory. Of course, this on its own is not a problem. It is going to be true of any deterministic theory that it is non-trivial to make sense of any other value of objective chance apart from 0 and $1 .^{2}$ Normally, this does not cause problems for confirmation. We can take the objective chances to be 0 or 1 and still be forced to rely on probabilistic evidence, or on relative frequencies, due to limitations in our epistemic situation. In the Everett interpretation we will often face a peculiar circumstance where the theory predicts with

\footnotetext{
${ }^{1}$ Throughout this article I will assume that we are talking about decoherence versions of Everettian quantum mechanics. Moreover, although I take all versions of Everettian theories to agree that all outcomes really occur, they do not all hold that they actually occur. In particular, Wilson [2013] has argued for a version of Everettianism where other branches should be given a modal interpretation as other possibilities rather than actualities.

${ }^{2} \mathrm{I}$ do not want to rule out that there are ways of retrieving non-trivial probabilities from deterministic theories. In particular best-system theories might be a way of doing this (see for example Hoefer [2007]).
} 
probability 1 that a certain outcome, or sequence of outcomes, will occur (in some branch or other) and also predicts with probability 1 that it will not occur (in some branch or other). At first glance, it looks like all that we can say about a sequence of outcomes that is allowed (but not necessitated) by the theory is that it definitely occurs in some branches and definitely does not occur in some branches. If this is really all that we can say about the probability of a sequence of outcomes, then it is hard to see how we could distinguish, among the sequences of outcomes that are allowed by the theory, which would count as confirmation for it and which would count as evidence against it. If we cannot makes sense of these claims, much of what we take to count as evidence in favour of other versions of quantum theory will fail to count as evidence for Everettian quantum theory. ${ }^{3}$

\subsection{The coherence problem, the quantitative problem, and the evidential problem.}

Greaves distinguishes three different problems related to the notion of probability in Everettian (or, many worlds) quantum theory. ${ }^{4}$ The coherence problem is the problem of making sense of ascribing any probability at all to outcomes of measurements. ${ }^{5}$ The quantitative problem is the problem of recovering probabilities in accordance with the Born rule. As Greaves and Myrvold note, the problem of making sense of probabilities within the many worlds theory is particularly pressing. Quantum mechanics is ordinarily understood as a theory that makes probabilistic predictions, and we take those predictions to be confirmed and disconfirmed in accordance with whether the outcomes (or statistical distribution of outcomes) that we see are ones that the theory tells us are relatively likely to occur. ${ }^{6}$ Greaves and Myrvold [2010] dub this problem the evidential problem and I will follow their terminology.

The overall picture of the problem is made slightly more complicated by the fact that a solution to the evidential problem does not have to involve a solution to the conceptual problem as it is stated above. The challenge of the evidential problem is to make sense of confirmation in such a way that the kind of statistical evidence that we take to count in favour of quantum mechanics continues to do so. Of course, we normally take those practices to involve a notion of non-trivial probabilities assigned to outcomes of experiments, but it is possible that there is some other, close enough, notion that could do the job. This means that we should rephrase the coherence problem somewhat. The

\footnotetext{
${ }^{3}$ I am much indebted to Greaves, Myrvold, Saunders, and Wallace for laying out and delineating these issues. ${ }^{4}$ See for example Greaves [2004, 3].

${ }^{5}$ That is to say, it is the question of how it can make sense to talk of any probability ascriptions to just the outcome of experiments rather than merely assigning 0 or 1 as the probability of the outcome occurring on some branch or other.

${ }^{6}$ That is, relative to the alternative hypotheses.
} 
truly pressing challenge is to have an Everettian way of making sense of something similar enough to the notion of non-trivial probabilities with respect to our practices of confirmation-including allowing probabilities (or something close enough) to be given by the Born rule. This is the project that I take the decision theoretic approach to take on.

\section{DeCision theOry to THE RESCue?}

The hope is that decision theory will be able to deliver something close enough to probabilities to provide a solution to both the new problem of coherence and the evidential problem. In order to have a solution to the evidential problem, we have to allow that probabilities (or something close enough) are given by the Born rule. There are two different prominent versions of how to tackle this problem. Very roughly, the strategy followed by Greaves and Myrvold is to show that a rational agent in a general, not specifically Everettian, branching situation acts as if she is maximising expected utility with respect to some probability function or other, and moreover that, given some further constraints, she acts as if she believes that there is some optimal such function about which she can learn (provided that she is not dogmatic). ${ }^{7}$ Greaves and Myrvold [2010, 287]—while not arguing that this is the uniquely rationally required probability measure - claim that merely taking it as a primitive of the theory that the probability measure is given by the Born rule at least leaves the many worlds interpretation in no worse a position than other versions of quantum mechanics and "no worse off than any other theory vis-à-vis the philosophy of probability".

... [C]onsider Everettian quantum mechanics as a theory that retains the Hilbert space framework, the same associations of operators with experimental set-ups and state vectors or density operators with preparation procedures, but replaces the Born rule with the rule: the squares of amplitudes are to be interpreted, not as chances of outcomes, but as branch weights. [Greaves and Myrvold, 2010, 284]

Wallace $[2012,151]$ accepts this position as an available back-up option but argues that Everettians can do better. His argument that Everettians can do better is the target of this article.

In order to show that Everettians can do better, Wallace elaborates on a theorem by Deutsch [1999]. The strategy is to start without making any assumptions about probability in an Everettian setting and to end by showing that a rational agent who both fully accepts Everettian quantum

${ }^{7}$ Greaves and Myrvold [2010] discuss in depth the considerations that should lead us to expect the rationality constraints to hold equally in a branching case as in an ordinary non-branching case. 
mechanics as true and who knows the state of her branch (from now on simply an Everettian agent for short) will take the Born rule to give the probability measure of future branches. This has not yet addressed the evidential problem, but Wallace [2012, chapter 6] argues that we can do so by relaxing the assumption that our agent is an Everettian one. The strategy is to first address the quantitative problem and to then use this in addressing the evidential problem. Crucially, for this strategy it is illegitimate to simply postulate that the chances are given by branch weights. This is what the solution to the quantitative problem aims to establish (and later use in addressing the evidential problem). The claim that Everettians can avoid just postulating that chances are given by branch weights in accordance with the Born rule is the way in which Everettians are claimed to do better than other quantum theories with respect to probability.

To argue against the claim that Everettians can do better, I will look in detail at a part of Wallace's derivation of the result that — without assuming anything probabilistic at the outset — we can derive that it is rationally required to take probabilities to be given in accordance with the Born rule. In particular, I will argue that the rationale for the initially plausible sounding principle of state supervenience is either unconvincing or not, as required, independent of probabilistic notions. ${ }^{8}$

\section{State Supervenience}

Wallace $[2010,238]$ describes the principle of state supervenience as a rationality constraint for Everettian agents. ${ }^{9}$ Informally he glosses the principle as below.

An agent's preferences between acts depend only on what physical state they actually leave his branch in: that is, if $U \psi=U^{\prime} \psi^{\prime}$ and $V \psi=V^{\prime} \psi^{\prime}$, then an agent who prefers $U$ to $V$ given that the initial state is $\psi$ should also prefer $U^{\prime}$ to $V^{\prime}$ given that the initial state is $\psi^{\prime}-U \succ^{\psi} V$ iff $U^{\prime} \succ^{\psi^{\prime}} V^{\prime}$.

Here, $\psi$ and $\psi^{\prime}$ give us the quantum states, and actions are represented by unitary operators $(U$, $U^{\prime}, V$, and $V^{\prime}$ ) on the states. $U \succ^{\psi} V$ is read as: at $\psi$ the agent prefers act $U$ to act $V$.

Wallace's [2010, 246] formal statement stays close to the informal one.

\section{State supervenience: If:}

\footnotetext{
${ }^{8}$ For challenges to other aspects of the argument see Dizadji-Bahmani [2015] for a challenge to branching indifference and a defence of branch counting, Baker [2007] for worries about the use of decoherence, and Adlam [2014] for challenges to the application of decision theory.

${ }^{9}$ The same formulation appears in Wallace $[2012,170]$. The formal version there contains a (very minor) typo, so I am quoting the earlier version here.
} 
- $\psi \in E$ and $\psi^{\prime} \in E^{\prime}$ for macrostates $E, E^{\prime}$;

- $U$ and $V$ are available at $E$, and $U^{\prime}$ and $V^{\prime}$ are available at $E^{\prime}$;

- $U \psi=U^{\prime} \psi^{\prime}$ and $V \psi=V^{\prime} \psi^{\prime}$

then $U \succ^{\psi} V$ iff $U^{\prime} \succ^{\psi^{\prime}} V^{\prime}$.

The only new notions that are explicitly mentioned here are, first, the idea of macrostates and, second, the availability of actions at macrostates. These are introduced since we are assuming that the agent does not care about the specific microstate. Instead, the agent cares only about where the agent is when it comes to the reward subspaces (of the particular quantum game). Finally, Wallace demands that the acts are available at $E$ (and $E^{\prime}$ respectively). Wallace [2010, 234] explains the need for this constraint since which acts are contemplatable may vary with the state.

3.1. State supervenience as a constraint on rationality. As a rationality constraint this principle is, at first glance, rather surprising. The name seems to indicate a metaphysical reading. The gloss that an agent's preferences between acts "depend only on what physical state they actually leave his branch in" is compatible with both a metaphysical and a normative reading in the context where it occurs. On a metaphysical reading, the dependence in question may be a metaphysical one. A metaphysical reading fits nicely with calling the principle a supervenience principle. Supervenience claims typically belong in metaphysics. However, against this is the fact that this principle occurs when Wallace $[2012,169]$ is listing "... rationality axioms". In this context, it is easy to read the claim as saying that a rational agent's preferences between acts depend only on what physical state they actually leave his branch in. Here the claim is normative. An agent's preferences between acts ought to only depend on the state they actually leave his branch in (on pain of irrationality). I will show that a metaphysical reading alone cannot make sense of the principle. The most plausible reading is that it is a rationality constraint that is supposed to follow from, in part, a metaphysical constraint.

3.1.1. Not a metaphysical constraint alone. In his discussion of Wallace's views, Saunders [2010, 25] initially favours a metaphysical reading, but gives a normative gloss to the statement.

But are his axioms reasonable? They are in part pragmatic constraints - constraints on the range and kind of acts that are available to an agent if the branching structure of the wavefunction is what Everettian quantum mechanics says it is. Another ('state supervenience') is an expression of physicalism: it says that an agent's preferences 
between acts should depend only on what state they leave his branch in. Others again are more overly rationalistic rules that are applicable more or less whatever the physical theory.

At first glance, a metaphysical reading fits nicely with Wallace's [2012, 171] positive arguments in favour of this principle. His first argument is brief.

A quick defence of state supervenience would be: the agent's preferences supervene on the actual state of the branch; transformations which differ only in how they would affect non-actual quantum states do not differ in any relevant respect.

Wallace is clear that he is committed to physicalism. This gives us that we expect the agent's preferences to supervene on the physical state that the agent is in. From this commitment it is not far to the conclusion that the agent's preferences metaphysically supervene on the actual state of the branch. The branch represents the complete state of the world that is capable of affecting the agent (since branches are relatively isolated). However, the physical state that the agent is in when we are considering the agent's preferences is not $U \psi, V \psi, U^{\prime} \psi^{\prime}$, or $V^{\prime} \psi^{\prime}$. $U \psi, V \psi, U^{\prime} \psi^{\prime}$, and $V^{\prime} \psi^{\prime}$ are the (post-branching) states that would result from the various actions that are being deliberated. The state of the branch (pre-branching) that the agent is in when we are considering the preferences of the agent are represented by $\psi$ and $\psi^{\prime}$. In so far as we are concerned about the agent's preferences supervening on the physical state of the agent, we expect the agent's preferences to supervene on $\psi$ and $\psi^{\prime}$. However, in the principle of state supervenience we are not assuming that $\psi$ and $\psi^{\prime}$ are the very same state. So nothing prevents it being true both-as physicalism plausibly demands - that the preference ordering of the agent supervenes on the state of the branch (pre-branching) and that the preference ordering in $\psi$ and $\psi^{\prime}$ differ from one another.

Wallace $[2012,166]$ is aware of this. When introducing the notation $U \succ^{\psi} U^{\prime}$, he is explicit that we need to index the preference ordering to the state of the agent's branch, $\psi$.

We now need to represent the agent's preferences between acts. Since those preferences may well depend on the state, we write it as follows: if the agent prefers (at $\psi$ ) act $U$ to act $U^{\prime}$, we write

$$
U \succ^{\psi} U^{\prime}
$$

If the principle of state supervenience is meant to follow merely from a metaphysical commitment to physicalism, then the reasoning fails. However, we may think that there is something close by 
that could do the job. Everettian quantum mechanics is a deterministic theory. From physicalism we get that the agent's preferences at $\psi$ supervene on $\psi$, and mutatis mutandis for the preferences at $\psi^{\prime}$. With the addition of determinism we know that there is a determinate outcome for each action over which the agent can have preferences. Although $\psi$ and $\psi^{\prime}$ are not assumed to be identical in the principle of state supervenience, we are assuming that $U \psi$ is identical to $U^{\prime} \psi^{\prime}$, and that $V \psi$ is identical to $V^{\prime} \psi^{\prime}$. However-from physicalism and determinism alone - it still does not follow that if $U \psi=U^{\prime} \psi^{\prime}$ and $V \psi=V^{\prime} \psi^{\prime}$, and the agent prefers $U$ to $V$ at $\psi$, then the agent also must prefer $U^{\prime}$ to $V^{\prime}$ at $\psi^{\prime}$. It is instructive to see why. From determinism we only get that each action at a state has a resulting state that is determined by the laws. If $U \psi=U^{\prime} \psi^{\prime}$ and $V \psi=V^{\prime} \psi^{\prime}$ entailed that $\psi=\psi^{\prime}$, then physicalism would deliver that the agent's preferences at $\psi$ and $\psi^{\prime}$ could not differ. However, determinism does not ensure that if $U \psi=U^{\prime} \psi^{\prime}$ and $V \psi=V^{\prime} \psi^{\prime}$, then $\psi=\psi^{\prime}$.

Physicalism alone does not deliver the principle of state supervenience. Neither does physicalism together with the fact that Everettian quantum mechanics is a deterministic theory. We need an additional assumption.

3.2. Metaphysical constraint plus a constraint on preferences. The principle of state supervenience cannot be defended merely by assuming physicalism and that Everettian quantum theory is deterministic. Wallace's $[2012,171]$ defence should lead us to suspect that this is the case. The claim was not, after all, that the initial, pre-branching, states do not differ, but that the outcome states do not differ "in any relevant respect". In the rest of this article I will spell out why I think that the assumption that they do not differ "in any relevant respect" cannot be defended without making assumptions about what probabilities are in Everettian quantum theory. This is a problem since Wallace will use the principle of state supervenience to try to establish that probabilities are given in accordance with the Born rule (for an Everettian agent) without making any assumptions about probability in Everettian quantum theory at the outset.

Within the claim that the outcomes do not differ in any way relevant to the agent's preferences between actions, there is an assumption that is not defended from physicalism or the determinism of Everettian quantum theory. This becomes clearer when Wallace $[2012,171]$ continues the defence of the principle of state supervenience. Since this is the totality of the defence, I include it here in full. 
... [T] his brings out a tacit assumption in the formalism: the idea that acts can be represented by single unitary transformations rather than by sequences of unitary transformations. Why regard a sequence of measurements as decision-theoretically equivalent to a single measurement just because the same unitary transformation is enacted by both?

One possible defence is that the agent is playing a sequence of games which result in rewards that he spends only after the sequence is done. In this case, what does he care about what happens during the brief period in which the games are being played (when having or not having rewards makes no difference to his status) should he not care only about the state of the universe after the payouts are all made?

However, appealing to intuition in this way - especially when discussing the Everett interpretation - is unsatisfactory. A far better defence is to observe that caring about the final state only is the diachronic equivalent of branch indifference, and can be defended in the same way. There is no 'real' branching structure beyond a certain fineness of grain, so the details of that structure can only be included in terms of their coarse-grained consequences.

The objection that Wallace is directly addressing is that the agent may care about how the final outcome came about. I will not pursue this objection. For the purposes of understanding the principle of state supervenience in this paper, the crucial assumption defended is that a rational Everettian agent cares only about the final state. Let us see how this could help in establishing state supervenience as an axiom of rationality. Here is a reconstruction of what I take to be a, at first glance, convincing argument. ${ }^{10}$

Definition: An Everettian agent knows the state of her branch and accepts Everettian quantum theory and physicalism.

Premise 1: Everettian quantum theory is true.

Premise 2: Physicalism is true.

Premise 3: A rational Everettian agent's preferences between actions reflect that the agent cares only about the final states (where rewards are paid out) resulting from actions.

\footnotetext{
${ }^{10}$ This way of arguing fits well with Wallace [2012, 2010, 2007] where Wallace starts arguing for routes to establish the principle of equivalence that do not go via a defence of the principle of measurement neutrality.
} 
Assumption 1: $U \psi=U^{\prime} \psi^{\prime}$ and $V \psi=V^{\prime} \psi^{\prime}$

Assumption 2: The rational Everettian agent prefers $U$ to $V$ at $\psi$.

Subconclusion 1: $U \psi, V \psi, U^{\prime} \psi^{\prime}$, and $V^{\prime} \psi^{\prime}$ fully capture the outcome of actions $U, V, U^{\prime}$, $V^{\prime}$ at $\psi$ and $\psi^{\prime}$, and the agent knows this. (from Definition and Premise 1 and 2)

Subconclusion 2: The rational Everettian agent at $\psi$ takes the state $U \psi$ to be better in terms of what she cares about than $V \psi$ (from Assumption 2, Premise 3, and Subconclusion 1)

Subconclusion 3: The rational Everettian agent at $\psi^{\prime}$ knows that $U \psi=U^{\prime} \psi^{\prime}$ and $V \psi=$ $V^{\prime} \psi^{\prime}$. (from Definition and Assumption 1)

Subconclusion 4: The rational Everettian agent at $\psi^{\prime}$ takes $U^{\prime} \psi^{\prime}$ to be better in terms of what she cares about than $V^{\prime} \psi^{\prime}$. (from Subconclusion 1, 2 and 3)

Conclusion: The rational Everettian agent prefers $U^{\prime}$ to $V^{\prime}$ at $\psi^{\prime}$. (from Subconclusion 4 and Premise 3)

The gist of the argument is straightforward (and seemingly compelling). If the agent cares only about the outcome of actions and knows what those outcomes will be, then there can be no relevant difference between a rational agent at $\psi$ and a rational agent at $\psi^{\prime}$ that would justify a difference in the preference rankings of actions with the same outcomes.

In the next section, I will argue that - in spite of the seeming plausibility of the argumentsubconclusion 4 does not follow from subconclusion 1,2 and 3. Further, the assumptions that are needed to motivate the move from subconclusion 1,2 and 3 to subconclusion 4 threaten to undercut the claim that the principle of state supervenience can be defended as a rationality constraint without making any specific claims about what probabilities are in Everettian quantum theory.

\section{Past EVIDENCE}

In this section I will present my worry about the principle of state supervenience as formulated by Wallace $[2012,2010]$. I will argue that we run into a dilemma.

Horn 1: We do not make probabilistic assumptions, and the principle of state supervenience is not defensible. 
Horn 2: We do make probabilistic assumptions, and the principle of state supervenience is defensible.

The principle is either not well-motivated as a rationality constraint on an Everettian agent's preferences, or it is unable to fulfil the argumentative role that Wallace puts the principle to. Remember, this principle is supposed to be a principle of rationality that Wallace $[2012,155-56]$ uses in order to provide " ... a fully rigorous argument that branch weight fills the functional role of objective probability, without any need to assume anything probabilistic at the outset".

4.1. The one world case. To get a more intuitive grip on the issues involved, I will start with the more familiar stochastic single world quantum case. The difference from the Everettian case is that $U \psi$ (etc.) no longer has to represent the final outcome of the action (where rewards are paid). Let us (with Wallace), for example, assume that the system is prepared in a superposition $\alpha|+\rangle+\beta|-\rangle$. Let us also assume that on action $U$, rewards are paid out if the measurement result is + but not if the result is - . The action $U$ now gives us $\alpha|+\rangle \otimes \mid$ reward $\rangle+\beta|-\rangle \otimes \mid$ no reward $\rangle$. In orthodoxy, we can read off the probability of the specific outcomes that the agent cares about-reward or no reward - from $U \psi$ by appealing to the Born rule. It is no longer true that $U \psi, V \psi$ (etc.) fully capture the outcome (that the agent cares about) of the action $U$ and $V$ at $\psi$. Rather, $U \psi$ captures information about the chances of various outcomes.

This already lands us in trouble. We need to assume that the chances supervene on $U \psi, V \psi$, etc. This is already a substantive assumption about chances and not mandated by physicalism (as we will see in section 4.3). For now, I will simply take on this assumption.

Let us now consider whether we can give a defence of state supervenience-without assuming the Born rule at the outset - for the one world quantum agent. I have stipulated that the agent knows that the objective chances of the various outcomes have to supervene on $U \psi$ and $V \psi$. Since the single world agent (just like her Everettian counterpart) also knows that $U \psi=U^{\prime} \psi^{\prime}$ and $V \psi=V^{\prime} \psi^{\prime}$, it is tempting to think that she is still forced to prefer $U^{\prime}$ to $V^{\prime}$ at $\psi^{\prime}$ if she prefers $U$ to $V$ at $\psi$. Let us stipulate that the agent only cares about the outcome of her action in so far as it is either $y_{1}$ or $y_{2}$. It looks irrational to accept both (1) and (2).

(1) The agent prefers action $A$ to action $B$. The agent knows that:

(a) action $A$ has chance $x_{1}$ of outcome $y_{1}$ and chance $x_{2}$ of outcome $y_{2}$.

(b) action $B$ has chance $x_{3}$ of outcome $y_{1}$ and chance $x_{4}$ of outcome $y_{2}$. 
(2) The agent does not prefer action $A^{\prime}$ to action $B^{\prime}$. The agent knows that:

(a) action $A^{\prime}$ has chance $x_{1}$ of outcome $y_{1}$ and chance $x_{2}$ of outcome $y_{2}$

(b) action $B^{\prime}$ has chance $x_{3}$ of outcome $y_{1}$ and chance $x_{4}$ of outcome $y_{2}$.

What is driving our judgement that an agent fitting both (1) and (2) is irrational is that we have stipulated that the agent knows what the chances of the various outcomes are on the actions considered. ${ }^{11}$ This is not trivially the case for the one world quantum agent. Indeed, that the agent knows what the chances are once the agent knows, say, $U \psi$, is exactly the kind of probabilistic assumption that a Wallace style argument needs to avoid.

At first, there may seem to not be much to this difference. After all, to see that the agent fitting both (1) and (2) has irrational preferences, I did not specify more than schematically what the chances are. What matter is that they are stipulated to be the same for both (1) and (2). In the case of state supervenience in the one-world setting, I am assuming that whatever the chances are (if there are any) they should (by the earlier assumption about chances supervening on $U \psi$, etc.) be the same for $U \psi$ and $U^{\prime} \psi^{\prime}$ since $U \psi=U^{\prime} \psi^{\prime}$ (and mutatis mutandis for $V \psi$ and $V^{\prime} \psi^{\prime}$ ). However, there is an important difference.

From the earlier stipulation, the agent knows that $U \psi$, etc. are the metaphysical basis for the chances of the various outcomes that she is interested in. It does not follow from this that she knows what those chances are. That is why orthodoxy specifies the Born rule. The agent should be committed to prefer $U^{\prime}$ to $V^{\prime}$ at $\psi^{\prime}$ if she prefers $U$ to $V$ at $\psi$ and knows that $U \psi=U^{\prime} \psi^{\prime}$ and $V \psi=V^{\prime} \psi^{\prime}$, only if she takes herself to have the same information about the chances of the outcomes that she cares about at $\psi$ and $\psi^{\prime}$. If she knew how to extract the chances from $U \psi$ and $U^{\prime} \psi^{\prime}$ (by, for example, the Born rule), then it would follow that she has the same information about the chances of the outcomes of the actions that she cares about at $\psi$ and at $\psi^{\prime}$. However, the assumption is that she does not know this. This is the type of probabilistic assumption that a Wallace style argument tries to derive by using the principle of state supervenience. It cannot be assumed.

To illustrate the importance of this, let me introduce an analogous toy case. Let us assume that we are flipping a genuinely stochastic coin. Let us also assume that physicalism is true. In particular, let us assume that the chances of the coin landing heads/tails supervenes on the exact

\footnotetext{
${ }^{11}$ There is an additional assumption needed: namely, this captures the total evidence that the agent has.
} 
set-up of the flip. Let us assume that coin flip set-up $A$ is identical to coin flip set-up $A^{\prime}$. The cost and the reward, should one be paid out, is equal in all bets. Let us also assume that our agent, Cyra, knows all of this.

(3) Cyra has observed the stochastic coin being flipped 1000 times. Every time the coin was prepared in set-up state $A$. 900 times it landed heads and 100 times it landed tails. Cyra is offered two bets. Bet $U$ will pay out iff the coin lands heads on the next flip when prepared in set-up state $A$. Bet $V$ will pay out iff the coin lands tails on the next flip when prepared in set-up state $A$. Cyra prefers bet $U$ to bet $V$.

(4) Cyra has observed the stochastic coin being flipped 1000 times. Every time the coin was prepared in set-up state $A^{\prime} .100$ times it landed heads and 900 times it landed tails. Bet $U^{\prime}$ will pay out iff the coin lands heads on the next flip when prepared in set-up state $A^{\prime}$. Bet $V^{\prime}$ will pay out iff the coin lands tails on the next flip when prepared in set-up state $A^{\prime}$. Cyra prefers bet $V^{\prime}$ to bet $U^{\prime}$.

Cyra knows that the chance of the coin landing heads on the next flip has to be the same in (3) and (4) since $A$ and $A^{\prime}$ are identical set-up states, and the chance supervenes on the set-up. Yet, there is nothing irrational about allowing Cyra's preference rankings as described in (3) and (4). Cyra knows that at most one of her preference rankings in (3) and (4) would be one that she would continue to endorse if she found out how to determine the chances from $A$ (and $A^{\prime}$ ). However, since she is not assumed to know this, it is not irrational for her to have one preference ranking in (3) while realising that had her evidence been different, as in (4), she would have had a different preference ranking. ${ }^{12}$

In our quantum case, the state that the agent's preferences may be taken to supervene on, $\psi$ and $\psi^{\prime}$, are not identical. As I argued above, there is no direct conflict with physicalism to suppose that the agent has different preference rankings in $\psi$ and $\psi^{\prime}$. Since the states are not identical, the agent can have different evidence about how to extract chances from $U \psi\left(U^{\prime} \psi^{\prime}\right)$ and $V \psi\left(V^{\prime} \psi^{\prime}\right)$ at $\psi$ and $\psi^{\prime}$. If we take horn 1 , and do not make any assumptions about how to get probabilities from $U \psi$, etc., then the principle of state supervenience does not look like a rationality constraint for the single world quantum agent. In orthodox quantum theories we postulate, as part of the theory, how to extract chances from $U \psi\left(U^{\prime} \psi^{\prime}\right)$ and $V \psi\left(V^{\prime} \psi^{\prime}\right)$ at $\psi$ and $\psi^{\prime}$; this is the work of the Born

\footnotetext{
${ }^{12}$ In particular, note that Cyra is not running afoul of the Principal Principle.
} 
rule. With this in place, state supervenience looks plausible, but we have made use of probabilistic assumptions.

So far I have reasoned about the principle of state supervenience only for one world quantum theories. Let me move on to consider the many worlds case.

4.2. The many worlds case. The reasoning in section 4.1 was based on the possibility of being uncertain about how to extract the chances of the alternative final outcomes from, say, $U \psi$, even under certainty that the chances supervene on $U \psi$. In Everettian quantum mechanics, and unlike orthodox quantum theories, $U \psi$ is the final outcome state. The principle of state supervenience is presented specifically as a rationality constraint for an Everettian agent. That is, an agent who accepts Everettian quantum mechanics and knows the state of her branch. I need to say more to show that state supervenience is not a rationality constraint for such an agent.

To do this, let us call our agent Everley. Everley accepts Everettian quantum mechanics as true and knows that $\psi$ represents the state of her branch. To make the case vivid and follow Wallace's $[2012,172]$ presentation, let us imagine that Everley is in the following scenario. Everley chooses between two acts $A$ and $B$. With Wallace, we will assume that Everley cares only about the final state (the outcome of her action) and only about that state in so far as the rewards are concerned. ${ }^{13}$

She knows that in each case a system is prepared in a superposition $\alpha|+\rangle+\beta|-\rangle$. Act $A$ results in state $A$, and mutatis mutandis for $B$, as below.

$$
\begin{aligned}
& A: \alpha|+\rangle \otimes \mid \text { reward }\rangle+\beta|-\rangle \otimes \mid \text { no reward }\rangle \\
& B: \alpha|+\rangle \otimes \mid \text { no reward }\rangle+\beta|-\rangle \otimes \mid \text { reward }\rangle
\end{aligned}
$$

That is, on act $A$ the reward is paid out on branches where the measurement is + while on act $B$ the reward is paid out on branches where the measurement is -. Here Everley does know, in one sense, what the outcome will be in terms of what she cares about. So far this seems like good news for the principle of state supervenience. Unlike the worry I presented in the one world case, Everettian Everley does not seem to need to extract chances about the final outcome from the unitary transformation since this now just $i s$ the final outcome. This joy is short lived. Even though she does know how the final state relates to rewards, she no longer has a trivial credence

\footnotetext{
${ }^{13}$ She does not, for example, care about how that final state was brought about, what the state is like at the microlevel, what the state is like at the macrolevel in terms other than the rewards, etc.
} 
(probability 0 or 1 ) that a certain action will produce a certain reward. This is, of course, no surprise. On Everettian quantum mechanics there is no single reward.

In fact, that there is no single reward is a crucial component in Wallace's argument. It is worth pausing here to remind ourselves of Wallace's [2012] argumentative strategy. Wallace [2012, 132] is providing what he calls a "rationalist approach" for understanding probability.

Frequentism, in identifying probability with relative frequency, fails (amongst other deficiencies) to reproduce its role in guiding action; an obvious alternative strategy to try is to regard probability's role in rational action as definitional and try to recover its connection with frequencies.

The strategy in [Wallace, 2012, 148] is to identify a feature of the world that comes to merit the name probability (or chance) through its role in guiding credences in decision making. Further, once we have recovered non-trivial decision guiding chances (or something close enough), Wallace [2012, 151] will try to show that "... arguments based on symmetry suffice to prove that the objective probabilities can be given only by the Born probability rule".

In the one world deterministic case, once all the information is known, there is no need for non-trivial credences.

For consider: the supposition is that if we knew all the relevant information, we would rationally judge a (fair) die to have probability $1 / 6$ of coming up six. But if we really knew all the information, then - since the die is deterministic - we would know precisely how it would land, and so would give six either probability zero or probability one. The symmetry is broken by the actual physical state of the die. Indeed, it could not be otherwise, since only one outcome occurs, so something must break the symmetry. [Wallace, 2012, 146]

Wallace's $[2012,150]$ suggestion is that in the many worlds case nothing breaks the symmetry, and nothing has to.

There is no analogous problem for the Everettian. There simply are no facts which could even in principle be available to an Everettian agent so that the agent knows his unique future, because there is no such unique future. [Footnote 25] ... I will suggest a possible metaphysics for worlds in which they are best thought of as differentiating one from another rather than splitting. On this theory, it is rather 
that each agent does have a unique future, but it is in principle impossible for him to possess reliable knowledge about that future.

For Wallace, it is crucial that although Everley does in one sense know how to connect the final state to rewards, she does not know what the outcome of an action will be in terms of rewards. If she knew what the unique reward of an action would be, then she would be in the case analogous to the deterministic die case. In the deterministic die case with full information, she has no need for non-trivial probabilities. That Everettian Everley does not know what the reward of an action will be allows Wallace to argue that she is forced to weight the various rewards that she is sure will take place. Here, however, is where the trouble lies.

Earlier I argued that the worry of the stochastic one world case - that our agent needs to extract chances from $U \psi$, etc.-may not apply in Everley's case. Now we have seen that in order to get non-trivial chances back, Wallace demands that she must do a decision theoretic weighting of all the reward outcomes that she knows will happen. The question is now whether Everley must, on pain of irrationality, do that weighting in such a way that she respects the principle of state supervenience. I think that the answer is no. Yet again, the states that the agent's preferences may be taken to supervene on, $\psi$ and $\psi^{\prime}$, are not identical. As I argued above, there is no direct conflict with physicalism to suppose that the agent has different preference rankings in $\psi$ and $\psi^{\prime}$. Since the states are not identical, the agent can also have different evidence about how to extract the weighting of the, now all occurring, reward outcomes from $U \psi, U^{\prime} \psi^{\prime}$ and $V \psi, V^{\prime} \psi^{\prime}$ at $\psi$ and $\psi^{\prime}$. As long as Everettian Everley is not stipulated to take a certain weighting to be the optimal one, or ascribe to a known rule for doing the weighting that is independent of $\psi$ and $\psi^{\prime}$-such as the Born rule - then we cannot make it a rational mandate for her to take the extraction made plausible at $\psi$ to be the same as the one made plausible at $\psi^{\prime}$.

When the "weights" were chances in the stochastic one world case, I argued that we have a dilemma. Either we assume nothing probabilistic, and the agent is not rationally compelled to accept Wallace's principle of state supervenience (Horn 1), or we do make probabilistic assumptions, and the agent may be rationally compelled to accept Wallace's principle of state supervenience (Horn 2). Since orthodox theories are happy to make probabilistic assumptions, they embrace the second horn without difficulty. For Wallace, however, this is a genuine dilemma. We cannot simply make the assumption that it is rationally mandated to take the Born rule to give the decision 
theoretic weights. This is what Wallace is trying to prove. Nor can we simply assume that it is rationally mandated that there is a rule for extracting the decision theoretic weighting that is independent of $\psi$ and $\psi^{\prime}$, and so makes the agent's preferences independent of $\psi$ and $\psi^{\prime}$. State supervenience (together with other rationality axioms) is supposed to prove this (see for example the equivalence lemma in Wallace [2012, section 5.7]).

Let us now return to our Everettian Everley. I will modify the case presented earlier. Let us stipulate that given the current state of her branch, $\psi$, Everley prefers act $A$ to act $B$. Earlier I represented act $A$ and act $B$ as resulting in the below.

$$
\begin{aligned}
& A: \alpha|+\rangle \otimes \mid \text { reward }\rangle+\beta|-\rangle \otimes \mid \text { no reward }\rangle \\
& B: \alpha|+\rangle \otimes \mid \text { no reward }\rangle+\beta|-\rangle \otimes \mid \text { reward }\rangle
\end{aligned}
$$

Let me now indicate explicitly that the result of the measurement done is up or down. Let me also explicitly indicate that the agent may have a record of past measurements that the new measurement is added to. I will represent the sequence of past measurements, available at $\psi$, by $\left|\operatorname{results}_{\psi}\right\rangle$.

$$
\begin{aligned}
& \left.\left.\left.\left.A: \alpha \mid \text { up }\rangle \otimes|+\rangle \otimes \mid \text { reward }\rangle \otimes \mid \text { results }_{\psi}\right\rangle+\beta \mid \text { down }\right\rangle \otimes|-\rangle \otimes \mid \text { no reward }\right\rangle \otimes \mid \text { results }_{\psi}\right\rangle \\
& \left.\left.\left.B: \alpha \mid \text { up }\rangle \otimes|+\rangle \otimes \mid \text { no reward }\rangle \otimes\left|\operatorname{results}_{\psi}\right\rangle+\beta \mid \text { down }\right\rangle \otimes|-\rangle \otimes \mid \text { reward }\right\rangle \otimes \mid \text { results }_{\psi}\right\rangle
\end{aligned}
$$

Now, let us also assume that had Everley's original state been $\psi^{\prime}$, then she would have been offered the analogous choice between actions $A^{\prime}$ and $B^{\prime}$ resulting in the states below.

$$
\begin{aligned}
& \left.\left.\left.\left.\left.\left.A^{\prime}: \alpha \mid \text { up }\right\rangle \otimes|+\rangle \otimes \mid \text { reward }\right\rangle \otimes \mid \text { results }_{\psi^{\prime}}\right\rangle+\beta \mid \text { down }\right\rangle \otimes|-\rangle \otimes \mid \text { no reward }\right\rangle \otimes \mid \text { results }_{\psi^{\prime}}\right\rangle \\
& \left.\left.\left.\left.\left.\left.B^{\prime}: \alpha \mid \text { up }\right\rangle \otimes|+\rangle \otimes \mid \text { no reward }\right\rangle \otimes \mid \text { results }_{\psi^{\prime}}\right\rangle+\beta \mid \text { down }\right\rangle \otimes|-\rangle \otimes \mid \text { reward }\right\rangle \otimes \mid \text { results }_{\psi^{\prime}}\right\rangle
\end{aligned}
$$

The actions are different from action $A$ and $B$ only in terms of being assumed to be available at state $\psi^{\prime}$. Since $\psi$ and $\psi^{\prime}$ are not the same state, it also follows that the record of past measurements at $\psi$ and $\psi^{\prime}$ may differ.

To take the reasoning further, I need to say a little about one of Wallace's richness axioms: Erasure. Wallace's $[2012,166]$ more informal statement of erasure postulates that "[g]iven a pair of states $\psi \in E$ and $\phi \in F$ in the same reward, there is an act $U$ available at $E$ and an act $V$ available at $F$ such that $U \psi=V \phi "$.

In our case $A$ and $A^{\prime}$ are alike when it comes to the rewards. Erasure then guarantees that there are act $A$-plus-erasure and $A^{\prime}$-plus-erasure available at $A$ and $A^{\prime}$ that result in state $A$-plus-erasure 
and $A^{\prime}$-plus-erasure such that $A$-plus-erasure $=A^{\prime}$-plus-erasure. The same reasoning applies to $B$ and $B^{\prime}$ so that $B^{\prime}$-plus-erasure $=B$-plus-erasure.

Everley is, by stipulation, indifferent as to the outcome of the measurement in itself (she cares only about the reward). Erasure makes use of this.

Erasure is slightly more complicated. It effectively guarantees that an agent can just forget any facts about his situation that don't concern things he cares about (i.e. by definition: that don't concern where in the reward space he is). In thinking about it, it helps to assume that any reward space has an 'erasure subspace' available (whose states correspond to the agent throwing the preparation system away after receiving the payoff but without recording the actual result of the measurement, say). [Wallace, 2012, 167]

Following Wallace's [2012, 172-73] reasoning, by making use of erasure and several other of Wallace's axioms we know that Everley is indifferent between the act $A$ and the act $A$-plus-erasure (and mutatis mutandis for $A^{\prime}, B$, and $\left.B^{\prime}\right) .{ }^{14}$

Let us stipulate that Everley prefers $A$ to $B$ at $\psi$. From this it follows - accepting the rationality constraints that I am not challenging here - that she also prefers $A$-plus-erasure to $B$-plus-erasure at $\psi$. If state supervenience is correct as a principle of rationality, then Everley should also prefer $A^{\prime}$-plus-erasure to $B^{\prime}$-plus-erasure at $\psi^{\prime}$. From this we can then get that she should prefer $A^{\prime}$ to $B^{\prime}$ at $\psi^{\prime}$.

This illustrates the crucial role that state supervenience plays in Wallace's argument. The step that I am challenging is the claim that, as a matter of rationality, Everley must prefer $A^{\prime}$-plus-erasure to $B^{\prime}$-plus-erasure at $\psi^{\prime}$ if she prefers $A$-plus-erasure to $B$-plus-erasure at $\psi$. I have already made the point in broad strokes above. This reasoning neglects to take into account the fact that the states $\psi$ and $\psi^{\prime}$ are different.

Note that I am not challenging that Everley is indifferent between performing $A$ and $A$-plus-erasure. To "hide" the past record available at $\psi$ is unproblematic when it comes to arguing that Everley is indifferent between $A$ and $A$-plus-erasure (since she, by stipulation, only cares about where she is in reward space), and we are dealing with Everley's preferences at $\psi$. The worry is that it is

\footnotetext{
${ }^{14}$ We are making use the rationality axioms of "branching indifference" and "diachronic consistency".
} 
not clearly legitimate to ignore the difference in the past record when we are comparing Everley's preferences at $\psi$ and $\psi^{\prime}$. Here the past record can differ.

In particular, if we do not stipulate that Everley is bound by a particular state-independent way of performing the decision theoretic weighting of the many outcomes, then it is plausible that she can take past evidence available at $\psi$ and $\psi^{\prime}$ into account when weighting the many reward outcomes. We are now back in a position similar to that of the one world case. Even if we take Everley to know that, as a matter of rationality, the optimal weighting must supervene on the final state (and, in the next section, we will see some reasons to doubt that this can be defended without probabilistic assumptions), she still only knows that the optimal weighting is, as a matter of fact, the same in $A^{\prime}$-plus-erasure and $A$-plus-erasure (similarly for $B^{\prime}$-plus-erasure and $B$-plus-erasure). If we do not stipulate that she has available to her what the correct weighting is - and we do not want to stipulate this - she can rationally hold both that the evidence at $\psi$ would lead her to favour a weighting such that she prefers $A$-plus-erasure to $B$-plus-erasure and that the evidence at $\psi^{\prime}$ would lead her to favour a weighting such that she prefers $B^{\prime}$-plus-erasure to $A^{\prime}$-plus-erasure. ${ }^{15}$

Of course, in the scenario above Everley also knows that at most one her preference rankings could be right about the optimal preference ranking (if there is one). However, there is nothing irrational about this as Cyra's case in section 4.1 illustrates.

We may think that it is impossible for Everley to not have a general and state independent method for extracting chances since she accepts Everettian quantum mechanics as a complete theory. However, this does not follow. Everettian quantum mechanics says nothing about chances. It only encodes information about branch weights. As someone who knows and accepts Everettian quantum theory, Everley is assumed to know the branch weights. However, these come to merit the name of chances only by the role that they, by the Wallace style argument, are taken to play in decisions by rational agents. ${ }^{16}$ Again, we cannot assume in defence of the principle of state supervenience that Everley has any particular rule for extracting chances (a decision theoretic weighting of the many real outcomes). The principle is supposed to be used in an argument

\footnotetext{
${ }^{15}$ For Wallace [2012] state supervenience plus the other axioms mentioned above are needed in order to first prove the equivalence lemma and then the Born rule. We cannot, therefore, stipulate the Born rule outright.

${ }^{16}$ When [Wallace, 2012, 249] is addressing the objection that probabilities in Everettian quantum theory seem to be subjective he responds that the "... quantum probabilities are objective ... they're relative branch weights. ... Although, branch weights are objective, what makes it true that branch weight = probability is the way in which the branch weights feature in the actions of (ideally) rational agents".
} 
that recovers the claim that the Born rule is the rationally mandated rule without making use of assumptions about probability in Everettian quantum theory. ${ }^{17}$

So far, my argument has remained general. Let me now consider a twist to the old worry about anomalous branches to illustrate how a difference in evidence for how to extract a weighting of outcomes at $\psi$ and $\psi^{\prime}$ from, say, $U \psi$ and $U^{\prime} \psi^{\prime}$ can play out. This will demonstrate the difficulty of providing an argument that a strategy that takes past evidence into account, even when the branch weight is known, is irrational. This is what we would have to be able to argue in order to establish the Born rule as rationally mandated for Everettian agents.

4.3. Everettian Everley and anomalous branches. Let us now stipulate that Everley finds herself on a branch that is assigned a low branch weight (in Wallace's sense). This is a branch where a sequence of outcomes that would be assigned a very low probability of occurring on orthodox quantum theories occurs. Everettian quantum mechanics guarantees that such branches exist. Without postulating what the chances (or the optimal decision theoretic weighting of the reward outcomes) are, I do not think that anomalous Everettian Everley is irrational in setting her credences to close to the observed frequencies. Putting the question in different terms, can we show that it would be irrational for Everettian Everley to do so, without assuming anything probabilistic at the outset?

Here is the case for the rationality of this Everettian Everley. With Wallace, Everley can adopt a functional definition of probability where anything that can play the Principal Principle role merits the name of objective chance. Moreover, whatever the chances are, they have to be the kind of chances that are compatible with determinism. Finally, she can, with Wallace, take the chances to be defined from an anthropocentric perspective. In an inessential, but simplifying, assumption let us take Everettian Everley to adopt the metaphysics that Wallace suggests above where she is uncertain about facts about her unique future. In some cases she will observe a stochastic looking pattern when she records measurement results, even though the underlying theory is deterministic. To be specific, let us say that Everley observes a pattern where 1000 measurements in the +, - basis of the state $\frac{1}{\sqrt{2}}|+\rangle+\frac{1}{\sqrt{2}}|-\rangle$ yields +900 times and -100 times. Given the Everettian background, the existence of this pattern is explicable and guaranteed by the structure of the set-up. Moreover, let us stipulate that Everley takes the higher level pattern of observed frequencies to be the best

${ }^{17}$ I believe that a similar worry can be raised against Sebens and Carroll's [forthcoming] principle ESP-QM if it is taken as a rationality constraint. 
guide to the world that there is in the Everettian setting, and she sets her credence in observing + on the next measurement to 0.9. We can even push this further and note that higher-level patterns like this one fit everything that best system theories with non-trivial chances under determinism, such as Hoefer's [2007], seem to demand from chances that should be guiding Everettian Everley's credences. The chances would now not supervene on $U \psi$, etc., but they do supervene on the physical: they merely do not supervene only on the outcome states. These chances are, in Hoefer's [2007, 585] words, “... g guide to life when you cannot find a better one ...". Everettian Everley can rationally hold that she cannot find a better one.

In particular, Everettian Everley may compare herself favourably to a Wallace Everettian agent. When Everettian Everley is on a branch that is assigned high branch weight, her credences will tend to coincide with those of Wallace's agents. When she finds herself on an anomalous branch, one that has a pattern of outcomes that does not get assigned a high branch weight, this pattern will be the best guide to life available. After all, this is a branch where the outcomes are not assigned a high branch weight. On such a branch she is better served by not letting branch weights guide her credences.

If she follows this strategy, then she will not respect the principle of state supervenience. Her preferences between actions in an Everettian setting supervene on the physical, but they do not supervene only on the physical state that they leave her branch in.

There is a tempting objection to give at this point.

4.3.1. Objection. I claimed above that an agent on an anomalous statistics branch is better served by not letting branch weight guide her credence. Here we may want to object that of course an agent actually on an anomalous statistics branch is better off by not letting branch weight guide credence. However, if we cannot know, or even have reliable information, that we are on an anomalous branch, then this is an unimplementable strategy and so one that Wallace [2010, 239] rejects as irrational.

$[\mathrm{W}]$ hat would it even be for a strategy to be rational, but physically impossible? By that token, the rational strategy for a trader is 'always buy shares that are going to increase in value'.

In one sense, the strategy that I have suggested is obviously not impossible to implement since the practice of using past frequencies as a guide to chances is what Wallace too suggests that we do when we do not know what the branch weights are. However, it may be objected that following this 
strategy will leave the agent worse off when she knows the quantum state than she would otherwise have been. Here we can grant that it would be best not to let branch weight guide credences on anomalous statistic branches if there is a reliable strategy available for doing so.

The worry of the objector is the following; when we are relying on past evidence, we know that we do not have full information about the degree to which our branch (including the yet unobserved portion) is anomalous. To imagine an analogous case, we can think of a fair coin that is flipped 1000 times. If the coin has landed heads 1000 times, then we may suspect that the coin is not fair. But if we are sure that the coin is fair, then we should not take it to be more probable that the coin will land heads in the 1001 flip than that it will land tails. If we could find out that we are part of a statistically anomalous sequence of fair coin flips that will yield an improbably high frequency of heads on future flips, then we should not set our credences in heads according to the chances dictated by the coin being fair (we have inadmissible information). However, in the absence of such information about future outcomes, and when we are sure that the coin is fair, then we should not increase our credence in the coin landing heads at the next flip. Similarly, although I should not set my credences according to the branch weights if I had information that my (unique) branch would be anomalous in the future, I can never have such information. In the absence of such information, when I find myself with statistics that show my branch to be anomalous in the past, I cannot use this to raise my credence that my branch will be anomalous in the future when I know the state of my branch and accept Everettian quantum mechanics as true. And it is the future outcomes that are relevant.

I think that this reasoning is right as far as it goes. However, it only gets its hold if we assume that the chances are given by the branch weights. In order to motivate the coin flip case, we had to assume that we are sure that the coin is fair. That is, we already have information about the chances. Otherwise, it would be rational to put the credence that the next flip will be heads much higher than 0.5. If I already take chances to be given by the branch weights, then I can explain why anomalous Everettian Everley's reasoning should be rejected. However, this strategy is not available to use when we are trying to give "... a fully rigorous argument that branch weight fills the functional role of objective probability, without any need to assume anything probabilistic at the outset". We have now yet again run up against the dilemma of section 4 . The goal was to recover probabilistic notions in general, and the Born rule in particular, by focusing on the preferences 
of a rational agent. This requires us to show that Everettian Everley is irrational. Yet, without making probabilistic assumptions at the outset, we cannot reject Everettian Everley's strategy as irrational.

\section{Conclusion}

I have argued against Wallace's claim that a rational Everettian agent must act as if she assigns probabilities in accordance with the Born rule. In particular, I have challenged Wallace's claim that the principle of state supervenience is a rationality constraint. Even if we accept the components of Wallace's positive arguments for this principle, they do not rule out an alternative principle that makes preferences between actions depend also on past evidence. This undermines the rationalist approach to recovering the Born rule.

While I have challenged the basis for Wallace's claim that the notion of probability is better understood in Everettian quantum theories than in one world theories, I have not directly argued against Wallace's claim that probability is no worse off in Everettian quantum mechanics.

Of course, the question remains: why on earth should Everettian agents condition their preferences between bets to conform with this mysterious 'branch weight' thing? And of course, Everettians who follow the primitivist line have no answer to give. To them, in effect, this requirement - the Principal Principle - is just a basic law of physics.

And to be sure, taking the Principal Principle as primitive is unattractive: why should it be a law of physics that I should care about branches in proportion to their weights? But we have already seen that this problem bedevils non-branching physics too: how can we accept any rationality principle as a law of physics? Or put the other way around, the suggestion that Everettians should indeed take this rationality principle as basic and unexplained ... seems on as solid ground as the analogous nonbranching suggestion. [Wallace, 2012, 150]

This back-up argument goes via simply postulating an Everettian version of the Principal Principle and taking it as primitive that chances are given by the branch-weights. Although I have not argued directly against this view, I have used an example of a best systems theory (Hoefer's [2007]) that fulfills many of the Everettian constraints on probability and that does not take the 
Principal Principle as primitive. If this is a plausible way of understanding probability, then we have an example where the notion of probability is better understood in one world settings. While this is, of course, not decisive, it does challenge the claim that the back-up primitivist strategy will clearly put Everettians on a par with one-worlders when it comes to the notion of probability.

\section{REFERENCES}

E. Adlam. The problem of confirmation in the Everett interpretation. Studies in History and Philosophy of Modern Physics, 47:21-32, 2014.

D. Baker. Measurement outcomes and probability in Everettian quantum mechanics. Studies in History and Philosophy of Modern Physics, 38:153-169, 2007.

D. Deutsch. Quantum theory of probability and decisions. Proceedings: Mathematical, Physical and Engineering Sciences, 455(1988):3129-3137, 1999.

F. Dizadji-Bahmani. The probability problem in Everettian quantum mechanics persists. British Journal for the Philosophy of Science, 66(2):257-283, 2015.

H. Greaves. Understanding Deutsch's probability in a deterministic multiverse. Studies in History and Philosophy of Modern Physics, 35(3):423-456, 2004.

H. Greaves and W. Myrvold. Everett and evidence. In A. Kent S. Saunders, J. Barrett and D. Wallace, editors, Many Worlds?: Everett, Quantum Theory, and Reality. Oxford University Press, 2010.

C. Hoefer. The third way on objective probability: A sceptic's guide to objective chance. Mind, 116(463):549-596, 2007.

S. Saunders. Many worlds? An introduction. In A. Kent D. Wallace S. Saunders, J. Barrett, editor, Many Worlds?: Everett, Quantum Theory, and Reality. Oxford University Press, 2010.

C. Sebens and S. Carroll. Self-locating uncertainty and the origin of probability in Everettian quantum mechanics. The British Journal for the Philosophy of Science, forthcoming.

D. Wallace. Quantum probability from subjective likelihood: improving on Deutsch's proof of the probability rule. Studies in History and Philosophy of Modern Physics, 38(2):311-332, 2007.

D. Wallace. How to prove the Born rule. In A. Kent D. Wallace S. Saunders, J. Barrett, editor, Many Worlds?: Everett, Quantum Theory, and Reality. Oxford University Press, 2010.

D. Wallace. The Emergent Multiverse: Quantum Theory according to the Everett Interpretation. Oxford University Press, 2012.

A. Wilson. Objective probability in Everettian quantum mechanics. British Journal for the Philosophy of Science, 64(4):709-737, 2013. 